This is a pre-print. The final publication is available at www.springerlink.com. Published in Bulletin of Mathematical Biology.

DOI: $10.1007 / \mathrm{s} 11538-011-9707-8$. 


\title{
Persistence in a single species CSTR model with suspended flocs and wall attached biofilms
}

\author{
Alma Mašićc ${ }^{a, b}$, Hermann J. Eberl ${ }^{c}$ \\ a) School of Technology, Malmö University, SE-20506 Malmö, Sweden \\ b) Centre for Mathematical Sciences, Lund University, Box 118, SE-22100, Lund, Sweden \\ c) Dept. Mathematics and Statistics, University of Guelph, ON, Canada \\ alma.masic@mah.se, heberl@uoguelph.ca
}

\begin{abstract}
We consider a mathematical model for a bacterial population in a continuously stirred tank reactor (CSTR) with wall attachment. This is a modification of the Freter model, in which we model the sessile bacteria as a microbial biofilm. Our analysis indicates that the results of the algebraically simpler original Freter model largely carry over. In a computational simulation study, we find that the vast majority of bacteria in the reactor will eventually be sessile. However, we also find that suspended biomass is relatively more efficient in removing substrate from the reactor than biofilm bacteria.
\end{abstract}

Keywords: biofilm, mathematical model, CSTR, wall attachment

MSC: 92D25

\section{Introduction}

Bacterial biofilms are microbial layers on immersed surfaces in aqueous systems. Bacteria adhere to the surface, become sessile and start producing a gel-like matrix of extracellular polymeric substances (EPS) in which they are themselves embedded [16]. Biofilms are omnipresent and can be found wherever environmental conditions sustain microbial growth. It has been suggested that the vast majority of bacteria in natural systems live in biofilm communities [9], and not in suspended communities, which have traditionally been the center of study, both in experimental microbiology and in mathematical biology. 
Two primary distinctions between the biofilm and the suspended mode of growth are (i) the EPS matrix offers the cells protection against mechanical washout and (ii) in a biofilm, the bacteria experience concentration gradients of dissolved substrates, i.e. the living conditions depend on the location of the cell within the biofilm $[23,26]$. The latter can lead to the establishment of micro-environments, such as anaerobic zones in aerobic biofilms $[8,26]$.

In environmental engineering many technologies have been developed based on biofilm processes, in particular in wastewater treatment, but also for soil remediation and groundwater protection [23]. Some of these technologies are based on stimulating biofilm growth by providing colonizable surfaces on which biofilms can form and be sheltered. An example of this kind are Moving Bed Biofilm Reactors (MBBR) for wastewater treatment, where the vessel in which the biological treatment processes occur is augmented by biofilm carrier chips [17].

In many engineered systems, including MBBR, biofilms and suspended communities co-exist. Moreover, free swimming bacteria may adhere to an already existing biofilm, and biofilm bacteria may leave the community and go into suspension. Both processes, attachment and detachment are not very well understood. The latter process is primarily associated with shear induced erosion or sloughing of biomass [19], but it has also been documented that cells can leave the community independently of external forces [6].

Mathematical models for suspended bacterial populations have been successfully developed for many years and are frequently and routinely used in design of reactors and operating conditions, and in the analysis of microbial growth curves, e.g. for food safety and shelf-life studies. Typically these are systems of first order ordinary differential equations. In microbially relatively simple systems, these models can often be studied analytically [24]. More complex microbial systems, e.g. the ones arising in wastewater treatment studies, usually must be studied computationally $[13,14]$.

Also mathematical models for biofilms have been developed for many years, originally primarily in the engineering literature $[29,30]$. They are more complex than models of suspended growth, because they must account for the spatial aspects of biofilms, most notably the inhomogeneous distribution of bacteria and substrates across the depth of a biofilm. Biofilm models are primarily computational models. Indeed, only very few studies are known in which biofilm models have been studied with analytical techniques [21, 27], although biofilm models have greatly contributed to our understanding of biofilm processes, in particular with respect to population and resource dynamics.

Mathematical models for systems that comprise both suspended and 
sessile bacteria are more scarce. Best known and well studied is the Freter model of competition between suspended and wall attached bacteria for a nutrient $[4,5,10,15,25]$. In this model, the amount of wall space available for colonization is limited and it is implicitly assumed that the wall attached bacteria form a relatively thin layer in which no concentration gradients develop. The cells sessile at the wall experience the same growth conditions as the suspended cells but are protected from hydrodynamical washout. Although this describes the system for which the model was originally developed (the mammalian gut) relatively well, it appears too simplistic for biofilm systems, where the wall attached bacteria can grow in thick layers in which concentration gradients can develop, and where the colonization surface is not limiting the capacity for wall attachment. A greatly simplified version of the Freter model has been used for description and analysis of gastro-intestinal $E$ coli infections [7].

In this study we revisit the Freter model for a CSTR. More specifically, we replace the original wall-attachment model by a traditional WannerGujer type one-dimensional biofilm model [29, 30], in order to account for the spatial structure of the wall attached bacterial population. The resulting model is algebraically more complex than the original Freter model and naturally the question arises whether this added algebraic complexity also adds new dynamic complexity. This is investigated with analytical and computational methods. Moreover, in a numerical simulation experiment we investigate how reactor operating conditions, such as flow rate, bulk substrate concentration, and surface area available for colonization affect the reactor's performance with respect to substrate removal.

\section{Model description}

\subsection{The Freter model of a CSTR with wall attachment}

In [10] a model was proposed that describes the formation of a microbial population in an aqueous environment in the mammalian gut. Bacteria can be suspended in the aqueous phase or colonize the surface. This model was later adapted to a CSTR [15], which includes the following processes:

- continuous replenishment of the reactor with fresh medium,

- washout of substrate and suspended biomass,

- growth of bacterial biomass through consumption of substrate,

- natural cell death, 
- attachment of suspended biomass to the wall,

- detachment of bacteria from the wall.

This is described by a system of three ordinary differential equations for the substrate concentration, the unattached as well as the wall-attached bacteria. The model reads

$$
\begin{aligned}
\dot{S} & =D\left(S^{i n}-S\right)-\gamma^{-1}\left(u \mu_{u}(S)+\delta w \mu_{w}(S)\right) \\
\dot{u} & =u\left(\mu_{u}(S)-D-k_{u}\right)+\beta \delta w+\delta w \mu_{w}(S)(1-G(W))-\alpha u(1-W) \\
\dot{w} & =w\left(\mu_{w}(S) G(W)-\beta-k_{w}\right)+\alpha u(1-W) \delta^{-1} .
\end{aligned}
$$

The dependent variables are concentration of the growth limiting substrate $S\left[\mathrm{gm}^{-3}\right]$, the concentration of suspended bacteria $u\left[\mathrm{gm}^{-3}\right]$, and the amount of biomass per unit area of colonizable surface, $w\left[\mathrm{gm}^{-2}\right]$.

The parameter $D\left[\mathrm{~d}^{-1}\right]$ is the dilution rate, i.e. the rate at which fresh substrate is supplied to the reactor, and at which substrate and suspended bacteria are washed out. It is obtained through $D=Q / V$ where $Q\left[\mathrm{~m}^{3} \mathrm{~d}^{-1}\right]$ is the flow rate and $V\left[\mathrm{~m}^{3}\right]$ the reactor volume. By $S^{i n}\left[\mathrm{gm}^{-3}\right]$ we denote the bulk concentration, i.e. the concentration at inflow. The constant $\delta\left[\mathrm{m}^{-1}\right]$ is a reactor parameter that denotes how much surface area is available for colonization per unit volume of the reactor.

The growth rates of the unattached and wall-attached bacteria are denoted by $\mu_{u}(S)$ and $\mu_{w}(S)$, respectively. The functional relationship between growth rate and substrate concentration is described by Monod kinetics, i.e. takes the form

$$
\mu_{u}(S)=\frac{m_{u} S}{a_{u}+S}, \quad \mu_{w}(S)=\frac{m_{w} S}{a_{w}+S},
$$

where the constants $m_{u}\left[\mathrm{~d}^{-1}\right]$ and $m_{w}\left[\mathrm{~d}^{-1}\right]$ are the maximum specific growth rates, i.e. the growth rates achieved in situations of food abundance, and the parameters $a_{u}\left[\mathrm{gm}^{-3}\right]$ and $a_{w}\left[\mathrm{gm}^{-3}\right]$ are the half saturation coefficients. The constant $\gamma[-]$ is the biomass yield per unit of substrate. The cell death rates are $k_{u}\left[\mathrm{~d}^{-1}\right]$ and $k_{w}\left[\mathrm{~d}^{-1}\right]$.

Daughter cells of the wall-attached bacteria compete for space; $G(W)$ cells attach to the wall at rate $\alpha\left[\mathrm{d}^{-1}\right], 1-G(W)$ are released into the bulk liquid, where $G(W)=\frac{1-W}{1.1-W}$ and $W:=w / w_{\max }$ is the wall occupancy fraction. Detachment of wall attached bacteria happens at rate $\beta\left[\mathrm{d}^{-1}\right]$.

It was found in [15] that the washout steady state $(S, u, w)=\left(S^{i n}, 0,0\right)$ always exists and that it is locally asymptotically stable if both $\mu_{w}\left(S^{i n}\right)-$ 
$k_{w}<0$ and $\mu_{u}\left(S^{i n}\right)-k-D<0$ are satisfied. It is unstable if either $\mu_{w}\left(S^{i n}\right) G(0)-k_{w}-\beta \geq 0$ or $\mu_{u}\left(S^{i n}\right)-k-D-\alpha \geq 0$ is satisfied. Further, the study showed that at least one nontrivial steady state $(S, u, w)=\left(S^{*}, u^{*}, w^{*}\right)$ exists when the washout state is unstable. However, given the complexity of the model, a calculation of the exact coordinates of the nontrivial steady state in closed form was not possible. Instability of the washout state implies persistence of $u$ and $w$, assuming nontrivial initial data.

\subsection{A CSTR model with wall attachment in form of biofilms}

In the model of [15] the wall attached bacteria experience the same substrate concentrations as the suspended bacteria. We will re-formulate the model for the case where the wall attached cells form bacterial biofilms. The starting point for our model is a CSTR mass balance as in the previous section, which we couple with a traditional one-dimensional biofilm model. The processes included in this model are the same as in Section 2.1. Additionally, we assume that

- the biofilm uniformly covers the surface that is available for colonization,

- substrate diffuses into the bacterial biofilm layer,

- substrate gradients in the biofilm are observed as a consequence of diffusion and reaction.

In the Freter model all sessile bacteria are assumed to be wall-attached and, therefore, compete for space. On the other hand, in a biofilm, sessile bacteria grow in thick layers that form on the substratum. In the WannerGujer model it is assumed that all available substratum is homogeneously covered. The fundamental underlying assumption of the traditional onedimensional biofilm model $[29,30]$ is that the biomass in a biofilm always attains maximum density. In a single-species biofilm, therefore, biomass and biofilm thickness are equivalent: production of biomass leads to a one-toone expansion of the biofilm. Biomass that is produced in the inner layers of the biofilm pushes the cells above, i.e biomass moves with a velocity that is equivalent to the rate of biomass production. The spatial extension of the biofilm distinguishes the new model from the Freter model. The consumption of substrate by the biofilm for growth induces a substrate flux across the biofilm/water interface. In the biofilm substrate diffuses and is degraded. This results in substrate gradients and, accordingly, the bacteria in the inner layers of the biofilm, where the substrate concentration is lower, 
live under different conditions than the bacteria closer to the interface, where the substrate concentration is higher. In particular, the bacterial growth rates in the biofilm are not homogeneous.

For our convenience, and unlike the model in Section 2.1, we cast the model in terms of the dependent variables substrate concentration $S\left[\mathrm{gm}^{-3}\right]$, suspended biomass $u[\mathrm{~g}]$ and biofilm thickness $\lambda[\mathrm{m}]$. The modified CSTR model with homogeneous biofilm wall attachment then reads

$$
\begin{aligned}
\dot{S} & =D\left(S^{i n}-S\right)-\frac{u \mu_{u}(S)}{V \gamma}-\frac{J(S, \lambda)}{V} \\
\dot{u} & =u\left(\mu_{u}(S)-D-k_{u}\right)+A \rho d(\lambda) \lambda-\alpha u \\
\dot{\lambda} & =v(\lambda, t)+\frac{\alpha u}{A \rho}-d(\lambda) \lambda
\end{aligned}
$$

Here, as above, $D$ is again the dilution rate, $S^{i n}$ the inflow concentration, $\gamma$ the yield coefficient, $k_{u}$ the cell death rate for suspended bacteria, and $\alpha$ is the rate at which suspended bacteria attach to the biofilm. In contrast to the model of the previous section, it does not depend on available wall space but models the attachment of suspended biomass to the biofilm.

In (4)-(6), $V\left[\mathrm{~m}^{3}\right]$ is the reactor volume and $A\left[\mathrm{~m}^{2}\right]$ the colonizable surface area. The biomass density in the biofilm is $\rho\left[\mathrm{gm}^{-3}\right]$.

In (6), the function $v=v(z, t)\left[\mathrm{md}^{-1}\right]$ denotes the growth induced velocity of the biomass at a location $z$ in the biofilm. Due to the incompressibility assumption that the biomass density is constant across the biofilm, biofilm expansion is essentially equivalent to biomass growth. Velocity $v$ is obtained as the integral of the biomass production rate

$$
v(z, t)=\int_{0}^{z}\left(\mu_{\lambda}(C(\zeta))-k_{\lambda}\right) d \zeta
$$

where $k_{\lambda}\left[\mathrm{d}^{-1}\right]$ is the cell death rate for biofilm bacteria.

Similarly as $\mu_{u}, \mu_{w}$ in Section 2.1, we define the substrate dependent growth rates via Monod kinetics, i.e.

$$
\mu_{u}(S)=\frac{\mu_{u}^{\max } S}{K_{u}+S}, \quad \mu_{\lambda}(C(z))=\frac{\mu_{\lambda}^{\max } C(z)}{K_{\lambda}+C(z)},
$$

where $\mu_{u}^{\max }, \mu_{\lambda}^{\max }$ are the maximum specific growth rates, $K_{u}, K_{\lambda}$ the halfsaturation coefficients and $C(z)\left[\mathrm{gm}^{-3}\right]$ denotes the substrate concentration in the biofilm at thickness $z[\mathrm{~m}]$ from the substratum. It is obtained as the 
solution of the two-point boundary value problem

$$
D_{c} C^{\prime \prime}(z)=\frac{\rho}{\gamma} \mu_{\lambda}(C(z)), \quad C^{\prime}(0)=0, C(\lambda)=S .
$$

Here $D_{c}\left[\mathrm{~m}^{2} \mathrm{~d}^{-1}\right]$ is the diffusion coefficient. The boundary condition at the substratum, $z=0$, describes that substrate does not leave the reactor through the walls, while the boundary condition at $z=\lambda$ implies that external mass transfer resistance at the biofilm/water interface is neglected. In (9) we used that substrate diffusion is a much faster process than biofilm growth, i.e. that (9) can be considered in a quasi-steady state.

In (4), the sink $J\left[\mathrm{gd}^{-1}\right]$ denotes the substrate flux from the aqueous phase into the biofilm, i.e.

$$
J(S, \lambda)=A D_{c} \frac{d C}{d z}(\lambda) .
$$

Detachment of biomass from the biofilm is described by the volumetric detachment rate $d(\lambda)\left[\mathrm{d}^{-1}\right]$. The frequently used detachment rate expression in biofilm modeling is to assume that $d$ is proportional to $\lambda$,

$$
d(\lambda)=E \lambda
$$

leading to a quadratic sink term in (6); $E\left[\mathrm{~d}^{-1} \mathrm{~m}^{-1}\right]$ is the erosion or detachment parameter.

We assume all model parameters to be positive.

\section{Analysis of the model}

In this section we present some analytical results about the equilibrium of the mathematical CSTR biofilms model. The following result shows that the model is well-posed and that the total mass in the system is bounded.

Proposition 3.1. Model (4)-(10) with nonnegative initial data

$$
S\left(t_{0}\right)=S^{0}, \quad u\left(t_{0}\right)=u^{0}, \quad \lambda\left(t_{0}\right)=\lambda^{0},
$$

possesses a unique, non-negative solution that exists for all $t>0$ and satisfies

$$
V \gamma S(t)+u(t)+A \rho \lambda(t) \leq a+b t
$$

where $a, b$ depend on model parameters and initial data. 
Proof. First we formally re-write our model as an ordinary initial value problem. Note that integrating (9) once and using the boundary conditions gives

$$
\frac{d C}{d z}(\lambda)=\frac{\rho}{\gamma D_{c}} \int_{0}^{\lambda} \mu_{\lambda}(C(z)) d z
$$

We define

$$
j(\lambda, S):= \begin{cases}\frac{\rho}{\gamma D_{c}} \int_{0}^{\lambda} \mu_{\lambda}(C(z)) d z, & \lambda>0 \\ 0 & \lambda=0 .\end{cases}
$$

Note that $C(z)$ is indirectly a function of $S$ due to the boundary condition in (9), therefore also $j$ is a function of $S$. Then (4)-(10) becomes

$$
\begin{aligned}
& \dot{S}=D\left(S^{i n}-S\right)-\frac{1}{V}\left(\frac{u \mu_{u}(S)}{\gamma}+A D_{c} j(\lambda, S)\right) \\
& \dot{u}=u\left(\mu_{u}(S)-D-k_{u}\right)+A \rho d(\lambda) \lambda-\alpha u \\
& \dot{\lambda}=\frac{\gamma D_{c}}{\rho} j(\lambda, S)-\lambda k_{\lambda}+\frac{\alpha u}{A \rho}-d(\lambda) \lambda .
\end{aligned}
$$

Some properties of the function $j(\lambda, S)$ are summarized in Lemma 3.3 below. In particular we note that $j(0, \cdot)=0$ and $j(\cdot, 0)=0$. Thus using the tangent criterion, see [28], it follows that the non-negative cone is positively invariant. Moreover, in the non-negative cone, the right hand sides of (16)-(18) are continuously differentiable, so the system satisfies a Lipschitz condition. This implies the local existence and uniqueness of a non-negative solution of the initial value problem with non-negative initial data.

Furthermore, adding the equations (16)-(18) gives the estimate

$$
V \gamma \dot{S}+\dot{u}+A \rho \dot{\lambda}=Q \gamma\left(S^{i n}-S\right)-u D-u k_{u}-A \rho \lambda k_{\lambda} \leq Q \gamma S^{i n} .
$$

Thus

$$
V \gamma S(t)+u(t)+A \rho \lambda(t) \leq V \gamma S^{0}+u^{0}+A \rho \lambda^{0}+Q \gamma S^{i n} t .
$$

Therefore, since $S, u, \lambda$ are non-negative, they exist and are bounded by a positive linear function for every $t>0$.

Remark 3.2. It is easily verified through eqs. (5) and (6) that all solutions $S, u, \lambda$ to the initial value problem (4)-(6) are such that either $u=\lambda=0$ or $u>0, \lambda>0$ for all $t$. Thus, if biomass is not absent from the system, it exists simultaneously in suspended and wall attached form. 
In the following lemma we derive a result that will be used later on in the stability analysis.

Lemma 3.3. The function $j(\lambda, S)$ for $\lambda \geq 0, S \geq 0$ is well-defined, nonnegative and differentiable. It has the following properties:

(a) $j(\cdot, 0)=0, j(0, \cdot)=0$,

(b) $\frac{\partial j}{\partial S}(0, S)=0$,

(c)

$$
\frac{S \theta}{K_{\lambda}+S} \leq \frac{\partial j}{\partial \lambda}(0, S) \leq \frac{S \theta}{K_{\lambda}}
$$

where $\theta=\frac{\rho \mu_{\lambda}^{\max }}{\gamma D_{c}}$ and $K_{\lambda}$ is the half-saturation coefficient from (8).

Proof. The function $j(\lambda, S)$ is well-defined since the boundary value problem (9) has a unique solution. If $\lambda$ and $S$ are positive, this solution is positive; since $C(z)$ is differentiable with respect to the parameters $\lambda$ and $S$, also $j(\lambda, S)$ is.

(a) For $S=0$ we have $C(\lambda)=0$ from the boundary conditions in (9). Since $C(z)$ is a continuous nonnegative function we have $C(z) \equiv 0 \Rightarrow$ $\mu_{\lambda}(C(z)) \equiv 0 \Rightarrow j(\cdot, 0)=0$.

(b) The derivative of $j$ with respect to $S$ for $\lambda=0$ is by definition

$$
\frac{\partial j}{\partial S}(0, S)=\lim _{h \rightarrow 0} \frac{j(0, S+h)-j(0, S)}{h} \stackrel{(a)}{=} 0 .
$$

(c) We non-dimensionalize (9) with $x=z / \lambda$ and $c(z)=C(z) / S$. The equation now becomes

$$
\begin{aligned}
& c^{\prime \prime}(x)=\lambda^{2} \frac{\rho \mu_{\lambda}^{\max }}{\gamma D_{c}} \frac{c(x)}{K_{\lambda}+S c(x)}, \\
& 0<x<1, \quad c^{\prime}(0)=0, c(1)=1 .
\end{aligned}
$$

Further, we consider the two linear auxiliary problems

$$
c^{\prime \prime}(x)=\theta \lambda^{2} \frac{c(x)}{K_{\lambda}+S}
$$


and

$$
c^{\prime \prime}(x)=\theta \lambda^{2} \frac{c(x)}{K_{\lambda}},
$$

with the same boundary conditions as in (20), where $\theta=\frac{\rho \mu_{\lambda}^{\max }}{\gamma D_{c}}$. The solution $c(x)$ of $(20)$ is continuous and bounded by $0<c(x)<1$. Therefore $K_{\lambda}<K_{\lambda}+c S<K_{\lambda}+S$. We denote the solutions to (21) and (22) by $c_{1}$ and $c_{2}$ respectively. With comparison theorems for Sturm-Liouville type boundary value problems, e.g. [28], we get

$$
c_{1}(x) \geq c(x) \geq c_{2}(x)
$$

for all $0<x<1$. Since $c_{1}(1)=c(1)=c_{2}(1)=1$ we conclude that

$$
c_{1}^{\prime}(1) \leq c^{\prime}(1) \leq c_{2}^{\prime}(1) .
$$

By reversing the non-dimensionalization, we obtain $c^{\prime}(1)=\frac{\lambda}{S} C^{\prime}(\lambda)$ and with (14) and (15) from (24)

$$
j_{1}(\lambda, S) \leq j(\lambda, S) \leq j_{2}(\lambda, S),
$$

where $j_{1}$ and $j_{2}$ are defined in analogy with (15) for the estimates $c_{1}$ and $c_{2}$. For a fixed $S, j_{1}(0, S)=j(0, S)=j_{2}(0, S)=0$ together with (25) gives us

$$
\frac{\partial j_{1}}{\partial \lambda}(0, S) \leq \frac{\partial j}{\partial \lambda}(0, S) \leq \frac{\partial j_{2}}{\partial \lambda}(0, S) .
$$

The solution to $(21)$ is

$$
c_{1}(x)=B_{1}(\lambda, S)\left(e^{x \sqrt{\theta_{1}(\lambda, S)}}+e^{-x \sqrt{\theta_{1}(\lambda, S)}}\right)
$$

with $\theta_{1}(\lambda, S)=\frac{\theta \lambda^{2}}{K_{\lambda}+S}$ and $B_{1}(\lambda, S)=\left(e^{\sqrt{\theta_{1}(\lambda, S)}}+e^{-\sqrt{\theta_{1}(\lambda, S)}}\right)^{-1}$. Similarly, the solution to (22) is

$$
c_{2}(x)=B_{2}(\lambda)\left(e^{x \sqrt{\theta_{2}(\lambda)}}+e^{-x \sqrt{\theta_{2}(\lambda)}}\right)
$$
with $\theta_{2}(\lambda)=\frac{\theta \lambda^{2}}{K_{\lambda}}$ and $B_{2}(\lambda)=\left(e^{\sqrt{\theta_{2}(\lambda)}}+e^{-\sqrt{\theta_{2}(\lambda)}}\right)^{-1}$. The fluxes are
now

$$
\begin{aligned}
& j_{1}(\lambda, S)=S B_{1}(\lambda, S) \sqrt{\frac{\theta}{K_{\lambda}+S}}\left(e^{\lambda \sqrt{\frac{\theta}{K_{\lambda}+S}}}-e^{-\lambda \sqrt{\frac{\theta}{K_{\lambda}+S}}}\right), \\
& j_{2}(\lambda, S)=S B_{2}(\lambda) \sqrt{\frac{\theta}{K_{\lambda}}}\left(e^{\lambda \sqrt{\frac{\theta}{K_{\lambda}}}}-e^{-\lambda \sqrt{\frac{\theta}{K_{\lambda}}}}\right)
\end{aligned}
$$


with

$$
\begin{aligned}
& \frac{\partial j_{1}}{\partial \lambda}(\lambda, S)=\frac{S \theta B_{1}(\lambda, S)}{K_{\lambda}+S}\left(e^{\lambda \sqrt{\frac{\theta}{K_{\lambda}+S}}}-e^{-\lambda \sqrt{\frac{\theta}{K_{\lambda}+S}}}\right) \\
& \frac{\partial j_{2}}{\partial \lambda}(\lambda, S)=\frac{S \theta B_{2}(\lambda)}{K_{\lambda}}\left(e^{\lambda \sqrt{\frac{\theta}{K_{\lambda}}}}-e^{-\lambda \sqrt{\frac{\theta}{K_{\lambda}}}}\right)
\end{aligned}
$$

which gives us

$$
\begin{aligned}
& \frac{\partial j_{1}}{\partial \lambda}(0, S)=\frac{2 S \theta B_{1}(0, S)}{K_{\lambda}+S}=\frac{S \theta}{K_{\lambda}+S}, \\
& \frac{\partial j_{2}}{\partial \lambda}(0, S)=\frac{2 S \theta B_{2}(0)}{K_{\lambda}}=\frac{S \theta}{K_{\lambda}} .
\end{aligned}
$$

The assertion follows from (26).

Remark 3.4. We observe that the estimates of $j(\lambda, S)$ are in fact

$$
\begin{aligned}
& j_{1}(\lambda, S)=S \sqrt{\frac{\theta}{K_{\lambda}+S}} \tanh \sqrt{\frac{\lambda^{2} \theta}{K_{\lambda}+S}}, \\
& j_{2}(\lambda, S)=S \sqrt{\frac{\theta}{K_{\lambda}}} \tanh \sqrt{\frac{\lambda^{2} \theta}{K_{\lambda}}},
\end{aligned}
$$

with $\theta=\frac{\rho \mu_{\lambda}^{\max }}{\gamma D_{c}}$ and $j_{1}(\lambda, S) \leq j(\lambda, S) \leq j_{2}(\lambda, S)$, where $j_{1}, j_{2}$ are the substrate fluxes of limiting first-order kinetic models. An illustration for $S=10 \mathrm{~g} / \mathrm{m}^{3}$ is given in Figure 1. An improved upper estimate for $j$ for small enough $\lambda$ could be obtained from the zero-order kinetics problem

$$
c^{\prime \prime}(x)=\frac{\theta S}{K_{\lambda}+S},
$$

which leads to a linear function in $\lambda$, see also [1].

Proposition 3.5. Let $\alpha>0$ and $d(\lambda)>0$. Then for the system of equations (4)-(6) the washout equilibrium $E_{0}=\left(S^{i n}, 0,0\right)$ exists for all parameters. It is asymptotically stable if

$$
\mu_{u}\left(S^{i n}\right)<D+k_{u}+\alpha \quad \text { and } \quad \frac{\partial j}{\partial \lambda}\left(0, S^{i n}\right)<\frac{k_{\lambda} \rho}{\gamma D_{c}}
$$




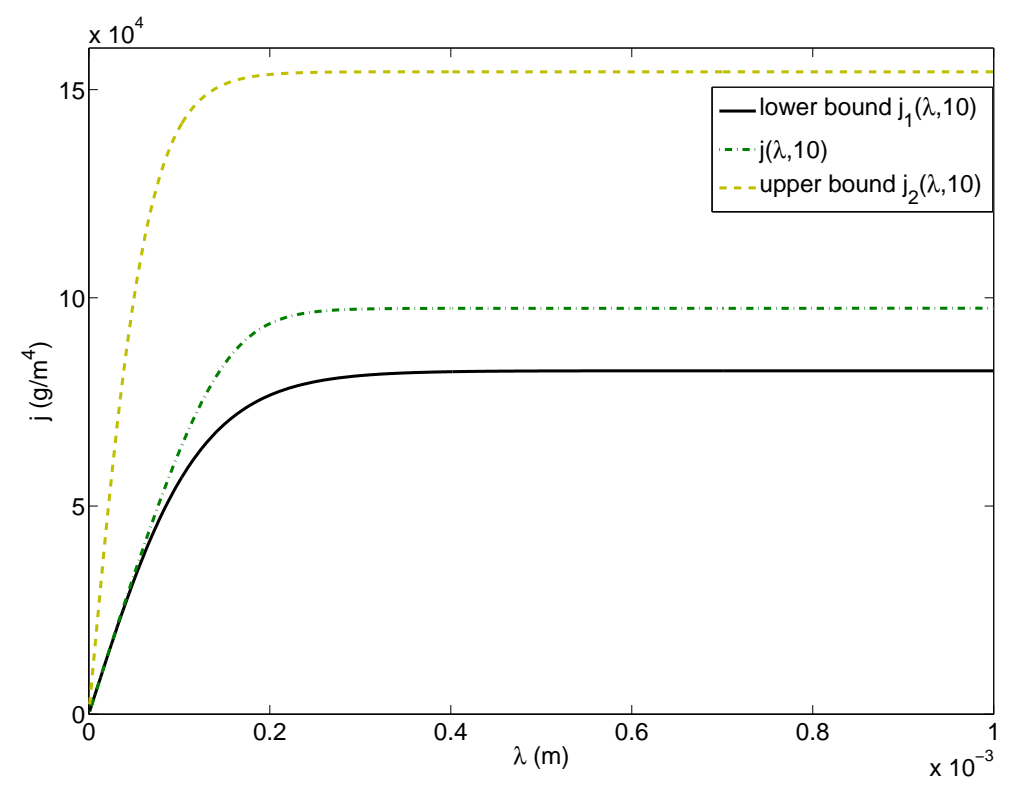

Figure 1: Numerical approximation of $j(\lambda, 10)$ with its upper (yellow dashed) and lower (black solid) bound using parameters from Tables 1 and 2. 
and unstable if either

$$
\mu_{u}\left(S^{i n}\right)>D+k_{u}+\alpha \quad \text { or } \quad \frac{\partial j}{\partial \lambda}\left(0, S^{i n}\right)>\frac{k_{\lambda} \rho}{\gamma D_{c}} .
$$

Proof. It is easily verified that the trivial equilibrium $E_{0}=\left(S^{i n}, 0,0\right)$ always exists. To determine the stability of the equilibrium we calculate the Jacobian $J(S, u, \lambda)$ of the right hand side of (16)-(18)

$$
\left(\begin{array}{ccc}
-D-\frac{u \mu_{u}^{\prime}(S)}{V \gamma}-\frac{A D_{c}}{V} \frac{\partial j}{\partial S}(\lambda, S) & -\frac{\mu_{u}(S)}{V \gamma} & -\frac{A D_{c}}{V} \frac{\partial j}{\partial \lambda}(\lambda, S) \\
u \mu_{u}^{\prime}(S) & \mu_{u}(S)-D-k_{u}-\alpha & A \rho\left(d(\lambda)+\lambda d^{\prime}(\lambda)\right) \\
\frac{\gamma D_{c}}{\rho} \frac{\partial j}{\partial S}(\lambda, S) & \frac{\alpha}{A \rho} & \frac{\gamma D_{c}}{\rho} \frac{\partial j}{\partial \lambda}(\lambda, S)-\left(d(\lambda)+\lambda d^{\prime}(\lambda)\right)-k_{\lambda}
\end{array}\right)
$$

which for $E_{0}$ simplifies to

$$
J\left(S^{i n}, 0,0\right)=\left(\begin{array}{ccc}
-D & -\frac{\mu_{u}\left(S^{i n}\right)}{V \gamma} & -\frac{A D_{c}}{V} \frac{\partial j}{\partial \lambda}\left(0, S^{i n}\right) \\
0 & \mu_{u}\left(S^{i n}\right)-D-k_{u}-\alpha & 0 \\
0 & \frac{\alpha}{A \rho} & \frac{\gamma D_{c}}{\rho} \frac{\partial j}{\partial \lambda}\left(0, S^{i n}\right)-k_{\lambda}
\end{array}\right) .
$$

The eigenvalues of $J\left(S^{\text {in }}, 0,0\right)$ are

$$
\begin{aligned}
\sigma_{1} & =-D, \\
\sigma_{2} & =\mu_{u}\left(S^{i n}\right)-D-k_{u}-\alpha, \\
\sigma_{3} & =\frac{\gamma D_{c}}{\rho} \frac{\partial j}{\partial \lambda}\left(0, S^{i n}\right)-k_{\lambda} .
\end{aligned}
$$

For asymptotic stability we need all eigenvalues to be negative. From this follows the assertion.

The function $j(\lambda, S)$ and its derivatives are not easy to evaluate. Instead, using the estimates from Lemma 3.3, we can derive the following weaker criterion

Corollary 3.6. A sufficient condition for asymptotic stability of the trivial equilibrium $E_{0}=\left(S^{\text {in }}, 0,0\right)$ is

$$
\mu_{u}\left(S^{i n}\right)<D+k_{u}+\alpha \quad \text { and } \quad \frac{S^{i n}}{K_{\lambda}}<\frac{k_{\lambda}}{\mu_{\lambda}^{\max }} .
$$

On the other hand,

$$
\mu_{u}\left(S^{i n}\right)>D+k_{u}+\alpha \quad \text { or } \quad \frac{S^{i n}}{K_{\lambda}+S^{i n}}>\frac{k_{\lambda}}{\mu_{\lambda}^{\max }}
$$

is sufficient for instability. 


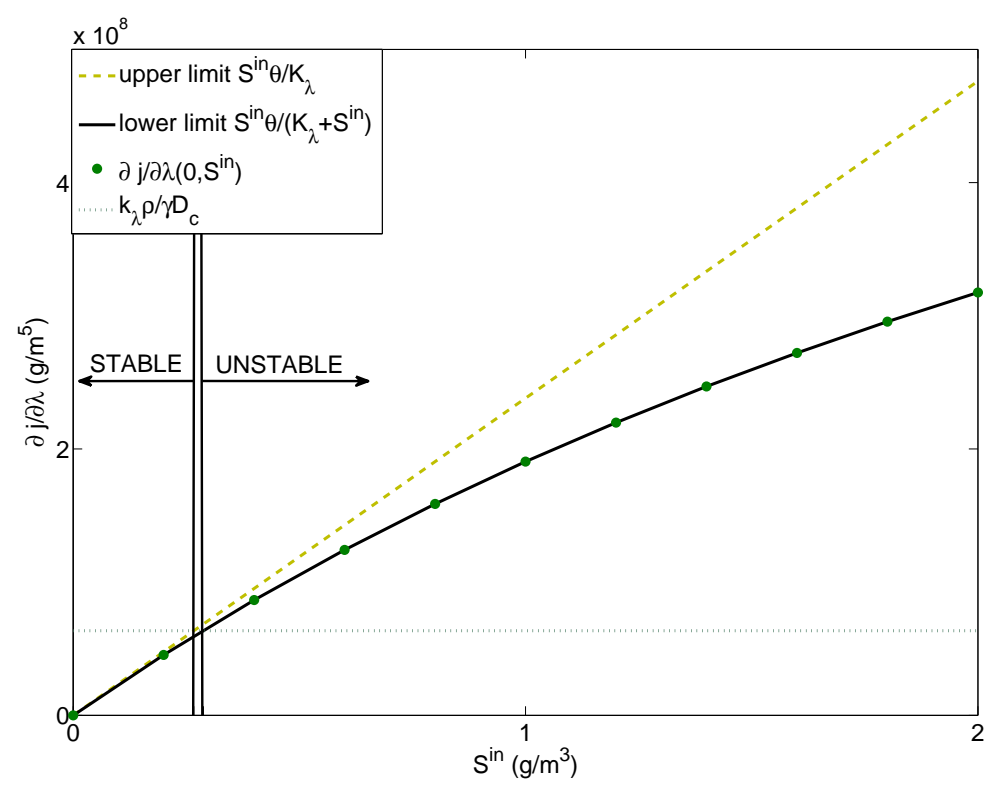

Figure 2: Numerical approximation of $\frac{\partial j}{\partial \lambda}\left(0, S^{i n}\right)$ (green dots) with its upper (yellow dashed) and lower (black solid) bound using parameters from Tables 1 and 2. The dotted line represents the constant $\frac{k_{\lambda} \rho}{\gamma D_{c}}$ from Proposition 3.5.

Observe that the stability criterion in Corollary 3.6 consists of two parts. One refers to the suspended biomass only and presents the classical persistence criterion where a population will not establish if the growth rate is smaller than the sum of dilution and death rate. The second criterion only refers to the biofilm: this stability and instability result is independent of the reactor flow rate and of the detachment rate coefficient $E$, in agreement with the analysis of [3].

Figure 2 illustrates the numerical approximation of $\frac{\partial j}{\partial \lambda}\left(0, S^{i n}\right)$ with its upper and lower estimate. We observe that the approximation of $\frac{\partial j}{\partial \lambda}\left(0, S^{i n}\right)$ corresponds to the lower estimate $\frac{\rho \mu_{\lambda}^{\max } S^{i n}}{\gamma D_{c}\left(K_{\lambda}+S^{i n}\right)}$. This is in good agreement with the results of [1], where an analytical approximation of the form $j(\lambda, S) \approx$ const $\cdot \frac{\lambda S}{K_{\lambda}+S}$ was derived with a Homotopy Perturbation Method argument, which could be numerically verified for an extended range of biofilm parameters. Stability of the trivial equilibrium $\mathrm{E}_{0}$ is attained for $S^{i n}$ such that $\frac{\partial j}{\partial \lambda}\left(0, S^{i n}\right)$ lies beneath $\frac{k_{\lambda} \rho}{\gamma D_{c}}$, which is represented by the dotted line in Figure 2.

In summary, with Proposition 3.1 we determine the existence of a unique, 
non-negative solution to our model (4)-(10), introducing the function $j(\lambda, S)$ in (15) and Lemma 3.3. Stability of the trivial equilibrium $E_{0}=\left(S^{i n}, 0,0\right)$ is determined in Proposition 3.5, where $E_{0}$ is asymptotically stable if $\mu_{u}\left(S^{i n}\right)<$ $D+k_{u}+\alpha$ and $\frac{\partial j}{\partial \lambda}\left(0, S^{i n}\right)<\frac{k_{\lambda} \rho}{\gamma D_{c}}$ are satisfied and it is unstable if either $\mu_{u}\left(S^{i n}\right)>D+k_{u}+\alpha$ or $\frac{\partial j}{\partial \lambda}\left(0, S^{i n}\right)>\frac{k_{\lambda} \rho}{\gamma D_{c}}$ is satisfied.

The stability conditions for $E_{0}$ obtained here are essentially the same as found in [15] for the Freter model with wall attachment. Since it was not possible to compute and analyze the non-trivial equilibria in the algebraically simpler Freter model, we did not attempt this here. Instead, we have investigated the model numerically in Section 4.

\section{Results}

\subsection{Computational details}

Numerical simulations of (4)-(6) were performed in Matlab with the builtin initial value problem solver ode15s. The embedded two-point boundary value problem (9) was solved with the routine bvp5c. The initial value problem was solved until a steady state was reached upon which the simulations were terminated. The initial conditions were

$$
S(0)=S^{i n} \mathrm{~g} / \mathrm{m}^{3}, \quad u(0)=10^{-6} \mathrm{~g}, \quad \lambda(0)=0 \mathrm{~m},
$$

where the reactor parameter $S^{\text {in }}$ was varied together with colonizable surface area $A$ and dilution rate $D$. This specific choice for $S(0)$ implies that the medium that is added to the reactor is the same as the medium with which the reactor is initially filled, which seems a reasonable assumption. Assuming a cylindrical reactor with dimensions specified in Table 1 we have a volume $V \approx 0.00118 \mathrm{~m}^{3}$ and an inner surface area $A_{\text {reactor }} \approx 0.055 \mathrm{~m}^{2}$. The surface area $A$ can be increased by adding suspended carriers on which biofilms can form [17], i.e. we always have $A \geq A_{\text {reactor }}$.

Table 1: Reactor dimensions.

\begin{tabular}{|l|c|c|}
\hline Parameter & Value & Reference \\
\hline radius & $0.05 \mathrm{~m}$ & assumed \\
height & $0.15 \mathrm{~m}$ & assumed \\
suspended carrier area & $0.0068 \mathrm{~m}^{2} /$ carrier & {$[17]$} \\
\hline
\end{tabular}

The model parameters are summarized in Table 2. To allow better comparison, we used the same growth parameter values for both biofilm and sus- 
pended biomass, i.e. we interpret the latter as microbial flocs, i.e. bacterial communities without substratum. The specific choices of model parameters are taken from Benchmark Problem 1 of the International Water Association's Taskgroup on Biofilm Modelling, i.e. our reactor models a sufficiently aerated system in which carbon is the only growth limiting substrate.

The values for the attachment rate $\alpha$ and erosion parameter $E$ were assumed. The erosion parameter $E$ was chosen large enough so that the results from our $1 \mathrm{D}$ model could be comparable to the results from a $2 \mathrm{D}$ model, as shown by [31]. In their study the authors investigate values ranging from $E=22.8 / d$ to $E=2280 / d$ and show that the biofilm thickness $\lambda(t)$ originating from a 1D model is very different from $\lambda(t)$ from a $2 \mathrm{D}$ model for small values of $E$ and very similar for large values of $E$. We picked a value in the middle of the range tested by these authors, which however can be viewed at the higher end of the range used by other authors. Of all the processes considered in our model, biofilm attachment is probably the least understood one. Also here we choose a relatively high value, assuming that one bacterium attaches to the wall for every six bacteria that grow in the bulk liquid.

Table 2: Model parameters.

\begin{tabular}{|c|l|c|c|}
\hline Symbol & Parameter & Value & Reference \\
\hline$\alpha$ & attachment rate & $1 /$ day & assumed \\
$D_{c}$ & diffusion coefficient & $10^{-4} \mathrm{~m}^{2} /$ day & {$[30]$} \\
$E$ & erosion parameter & $1000 /(\mathrm{m} \cdot$ day $)$ & assumed \\
$\gamma$ & yield of biomass from substrate & $0.63-$ & {$[30]$} \\
$K_{\lambda}, K_{u}$ & half-saturation coefficients & $4 \mathrm{~g} / \mathrm{m}^{3}$ & {$[30]$} \\
$k_{\lambda}, k_{u}$ & death rates & $0.4 /$ day & {$[30]$} \\
$\mu_{\lambda}^{\text {max }}, \mu_{u}^{\text {max }}$ & maximum specific growth rates & $6 /$ day & {$[30]$} \\
$\rho$ & biofilm biomass density & $10000 \mathrm{~g} / \mathrm{m}^{3}$ & {$[30]$} \\
\hline
\end{tabular}

\subsection{Typical simulations}

We solved (4)-(10) numerically, stopping the simulations once the solutions had attained steady state. In accordance with our analysis, two steady state forms were observed, depending on initial substrate concentration and dilution rate: complete washout of all biomass and co-existence of both biomass types, suspended and biofilm. Variation of the initial suspended 

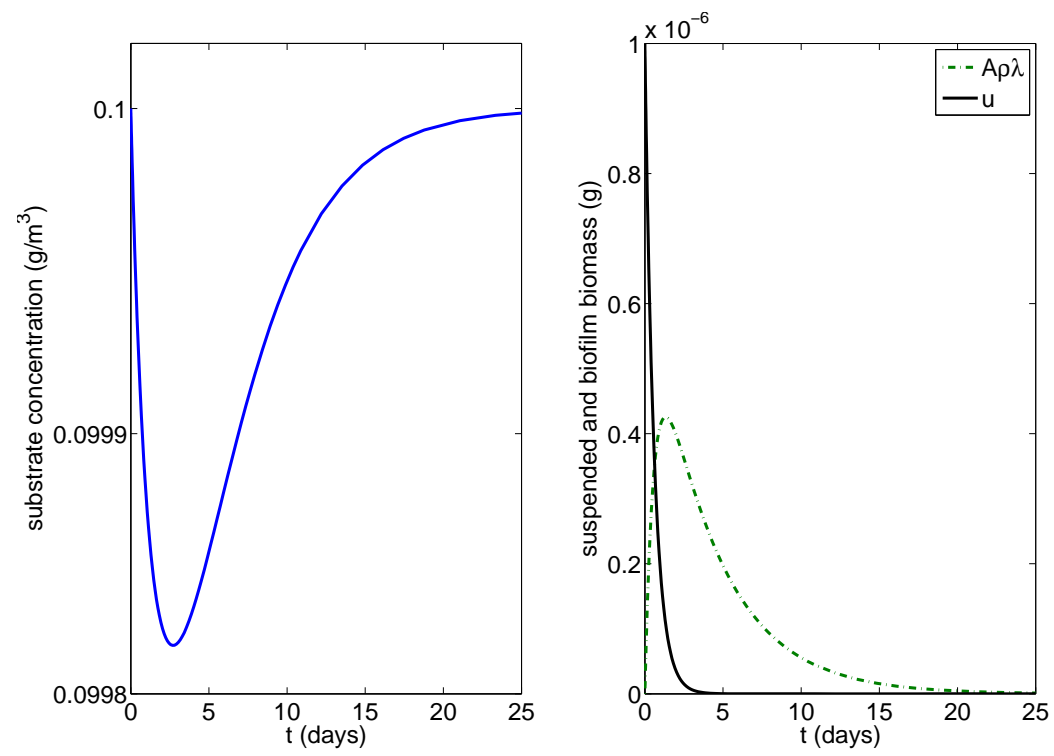

Figure 3: Typical time-dependent simulation at $D=0.42 /$ day and $S^{0}=0.1$ $\mathrm{g} / \mathrm{m}^{3}$ with substrate concentration $S$ (left) and suspended biomass $u$ and biofilm biomass $A \rho \lambda$ (right).

biomass $u^{0}$ did not have an impact on the steady state values.

For a small initial substrate concentration $S^{0}=0.1 \mathrm{~g} / \mathrm{m}^{3}$ and a small dilution rate $D=0.42$ /day we observed washout, see Figure 3 . Here $D$ was much lower than the maximal growth rate $\mu_{\lambda}^{\max }=\mu_{u}^{\max }=6 /$ day, wherefore we expected little biomass to be flushed out of the reactor, compared to the amount of suspended biomass being produced. However, the initial substrate concentration was also very low and the slow dilution rate did not supply much substrate with the influent. Even though biofilm initially started growing it was limited by the low substrate concentration, eventually being completely washed out of the reactor. The suspended biomass started at $u^{0}=10^{-6} \mathrm{~g}$ and decreased toward zero. The low initial substrate concentration in combination with the dilution rate sufficed to wash out all biomass from the reactor.

By increasing the bulk substrate concentration $S^{\text {in }}$, we expected the biomass to have access to enough nutrients to persist. Keeping the same dilution rate and increasing $S^{0}$ to $10 \mathrm{~g} / \mathrm{m}^{3}$, we observed biomass growth and co-existence. Both biomass types increased until they reached a steady state while the substrate was consumed, see Figure 4. The steady state 

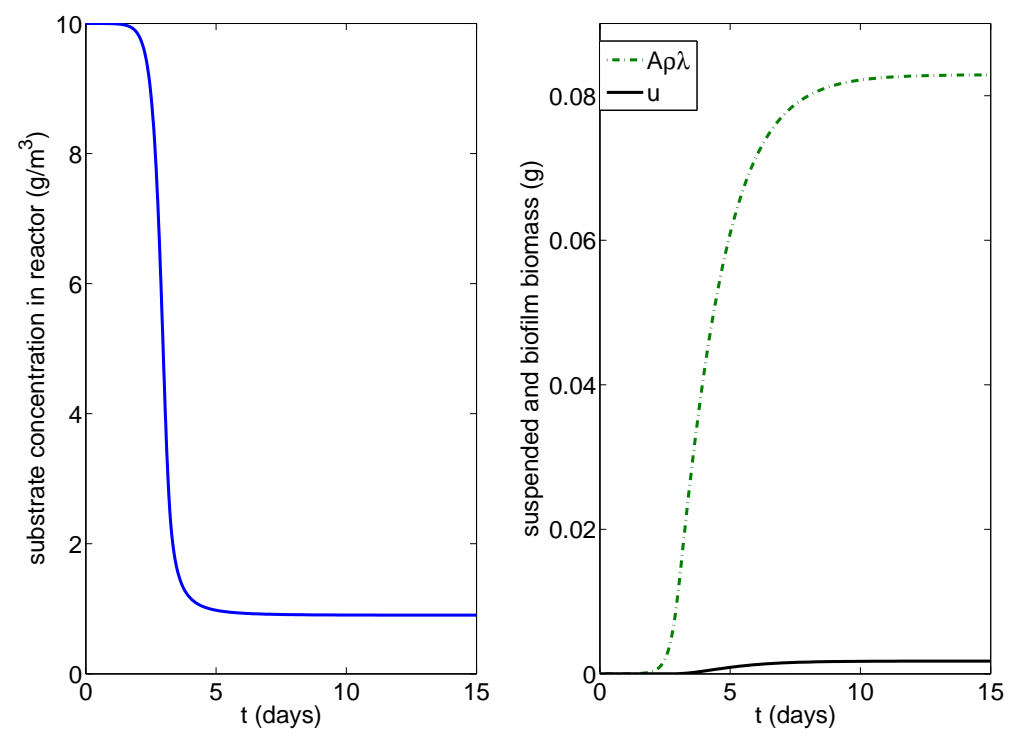

Figure 4: Typical time-dependent simulation at $D=0.42 /$ day and $S^{0}=10$ $\mathrm{g} / \mathrm{m}^{3}$ with substrate concentration $S$ (left) and suspended biomass $u$ and biofilm biomass $A \rho \lambda$ (right).

values indicate prevalence of biofilm biomass.

\subsection{Trivial equilibrium}

Using the estimates from Corollary 3.6 and the parameters from Table 2 we know that asymptotic stability of the trivial equilibrium $\mathrm{E}_{0}=\left(S^{i n}, 0,0\right)$ is achieved if $S^{i n}<0.2667 \mathrm{~g} / \mathrm{m}^{3}$ and instability if $S^{i n}>0.2857 \mathrm{~g} / \mathrm{m}^{3}$. The nature of the interval between these two extremal values is unknown and cannot be determined with the estimates. Therefore, we performed numerical simulations for $S^{\text {in }} \in[0.26,0.29] \mathrm{g} / \mathrm{m}^{3}$ at the relatively high dilution rate $D=85 / d \gg 6 / d=\mu$ to investigate the interval in more detail. A close-up of the interval is presented in Figure 5 with steady state values of the solutions. Both biomass types were washed out for the lower values of $S^{i n}$. The critical value of $S^{\text {in }}$, when both suspended and biofilm biomass are positive, was in fact closer to $0.2857 \mathrm{~g} / \mathrm{m}^{3}$, given by the upper bound in Corollary 3.6.

Led by the conclusions of Proposition 3.5 we were interested in the behavior of our model for variations in parameters $D$ and $S^{i n}$. We rendered a coarse two dimensional grid and performed numerical simulations 


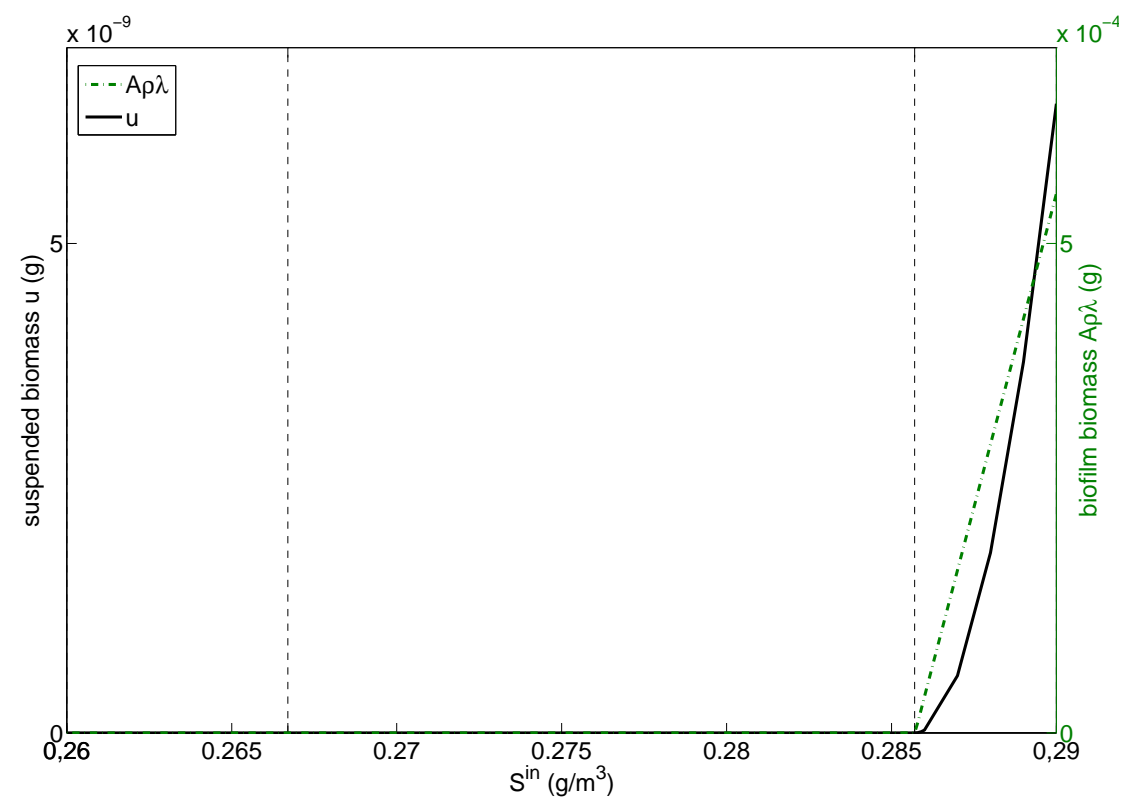

Figure 5: Steady state values of suspended and biofilm biomass, on two different axes, for $S^{\text {in }} \in[0.26,0.29] \mathrm{g} / \mathrm{m}^{3}$ at $D=85 /$ day, showing a close-up of $E_{0}$ becoming unstable. Two vertical dashed lines indicate the critical values given by Corollary 3.6. 

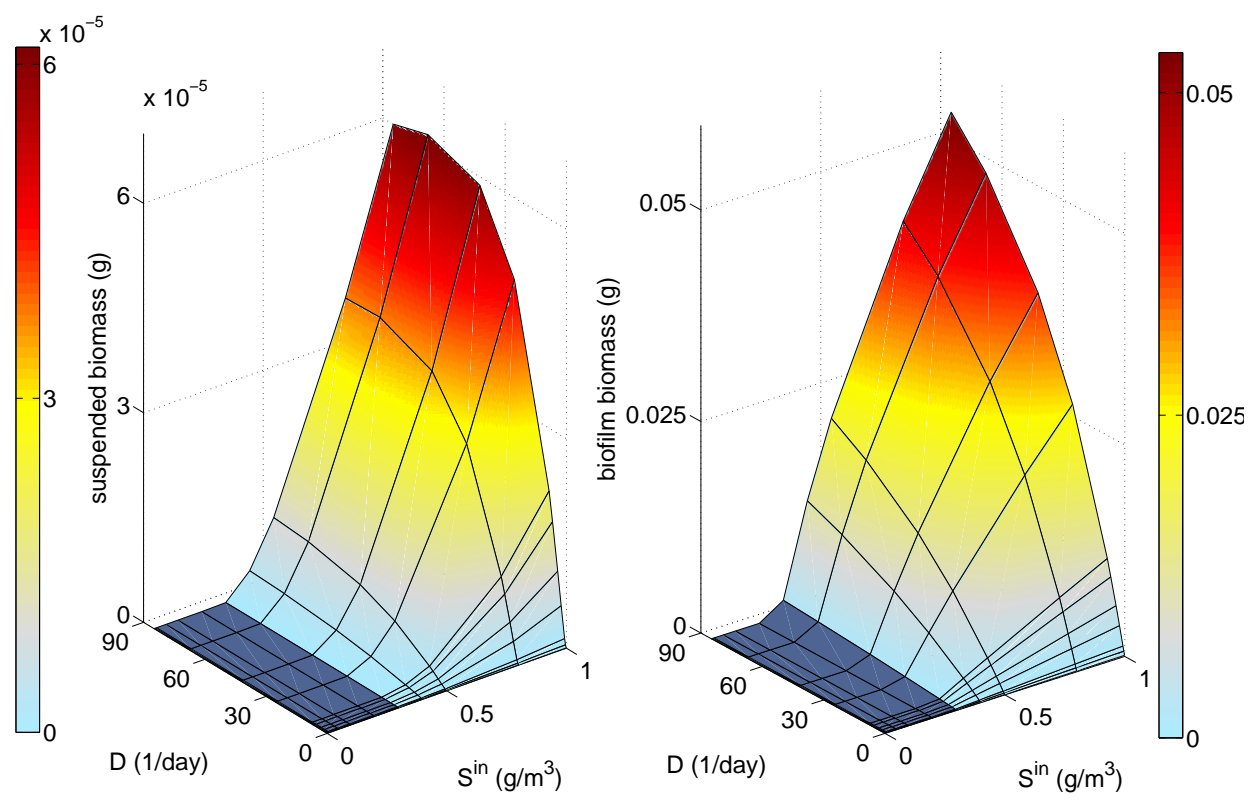

Figure 6: Suspended biomass $u$ (left) and biofilm biomass $A \rho \lambda$ (right) at steady state as functions of the dilution rate $D$ and the initial substrate concentration $S^{i n}$. Trivial equilibrium $E_{0}=\left(S^{i n}, 0,0\right)$ marked in dark blue.

for $0<S^{\text {in }} \leq 1 \mathrm{~g} / \mathrm{m}^{3}$ and $0<D<85$ /day, presented in Figures 6 and 7. We expected impact on the stability of the trivial equilibrium from both $D$ and $S^{i n}$, depending on the signs of the eigenvalues in Proposition 3.5. Our results, however, show that the stability was governed solely by $S^{i n}$. Washout of all biomass occurred for $S^{i n}<0.3 \mathrm{~g} / \mathrm{m}^{3}$, independent of the values of $D$. This is a value much smaller than the half saturation concentration, i.e. indicates a regime of nutrient scarcity. The critical value of $S^{\text {in }}$ for which the third eigenvalue $\sigma_{3}$ becomes positive is rather small. In this range of $S^{i n}$, the second eigenvalue $\sigma_{2}$ remains negative, implying that $\sigma_{3}$ is always the first eigenvalue to become positive as $S^{\text {in }}$ varies. Since $D$ is not a part of the expression for $\sigma_{3}$, the stability of $\mathrm{E}_{0}$ is governed by the reaction processes only, but does not depend on the bulk hydrodynamics. An increase in $S^{\text {in }}$ beyond the critical value caused instability of $\mathrm{E}_{0}$ expressed by an increase in biomass. 


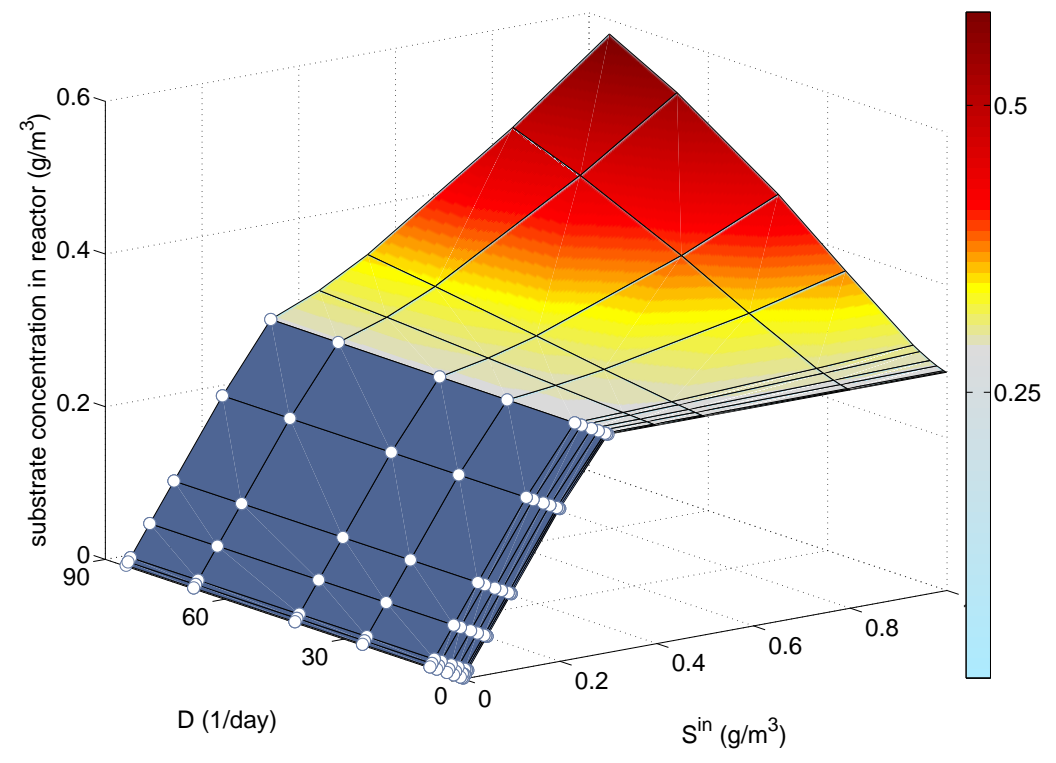

Figure 7: Substrate concentration $S$ in the reactor at steady state as function of the dilution rate $D$ and the initial substrate concentration $S^{i n}$. Trivial equilibrium $E_{0}=\left(S^{i n}, 0,0\right)$ marked in dark blue with white circles. 

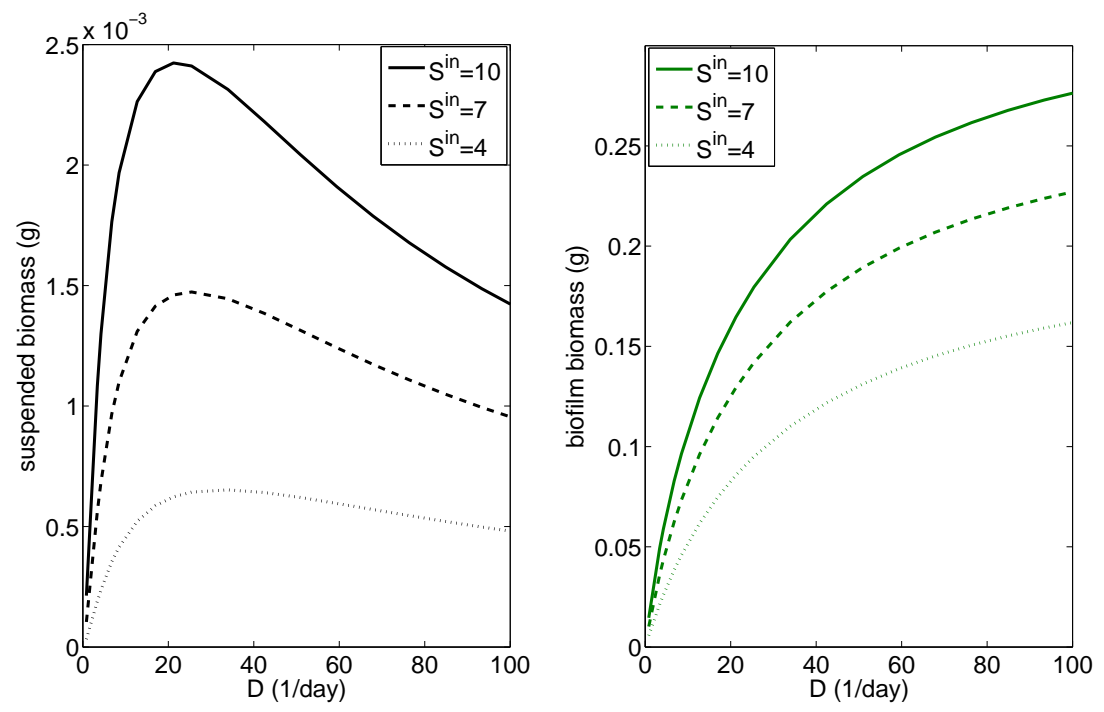

Figure 8: Suspended biomass $u$ (left) and biofilm biomass $A \rho \lambda$ (right) at steady state as functions of the dilution rate $D$ at $S^{i n}=10,7$ and $4 \mathrm{~g} / \mathrm{m}^{3}$.

\subsection{Persistence equilibrium}

We know from Proposition 3.5 that the trivial equilibrium is unstable, i.e. $S, u, \lambda>0$, when $S^{i n}$ is large enough. Therefore, the longterm behavior of the model was investigated numerically through variation of $S^{i n}$ and $D$. Washout occurred for $S^{\text {in }}<0.3 \mathrm{~g} / \mathrm{m}^{3}$, as previously discussed. Persistence of both biomass types was achieved in every simulation for $S^{i n} \geq 0.3 \mathrm{~g} / \mathrm{m}^{3}$. The two biomass types behaved differently as $S^{i n}$ and $D$ varied. Suspended biomass increased in the lower range of $D$ while the dilution rate was still comparable to the growth rate. But as $D$ rose to multiples of $\mu_{u}^{\max }=6 /$ day it acted as the stronger force, wherefore more suspended biomass was washed out before it could notably contribute to suspended growth. Eventually, for very large $D$, there was very little suspended biomass in the reactor, however never vanishing completely. An increase in $S^{\text {in }}$ increased the peak of suspended biomass before it declined due to washout. Figure 8 is a snapshot of steady states of $u$ and $A \rho \lambda$ at $S^{i n}=4,7$ and $10 \mathrm{~g} / \mathrm{m}^{3}$ as they vary along $D$. Biofilm biomass increased for both $S^{\text {in }}$ and $D$, eventually reaching a plateau for large $D$.

Figure 8 indicates that the suspended biomass will decrease eventually as $D$ increases, whereas the biofilm will persist and, in fact, keep growing. 
Larger $D$ brings more nutrients to the reactor wherefore the biofilm thickness increases. Since the detachment rate (11) has no connection to the dilution rate, it is not affected by variation in $D$. Eventually, suspended biomass will decrease to a minimum due to washout from the reactor for a large enough dilution rate.

In our model (4)-(6), the bulk flow rate contributes to substrate supply and to washout of suspended biomass. Experimental results indicate that also the rate of detachment is coupled to the bulk hydrodynamics, more specifically, that detachment forces increase as the bulk flow rate increases [19]. This is not reflected in the simple standard detachment model (11), which assumes that the detachment rate is proportional to the biofilm thickness only.

In order to investigate the possible contribution of the reactor flow rate to detachment and the effect that this has on the reactor behavior, we coupled the detachment rate $E$ to the dilution rate $D$. Motivated by [22] we chose the relationship as

$$
\tilde{E}=E\left(\frac{D}{D_{0}}\right)^{0.58},
$$

where by $D_{0}$ we denote a reference dilution rate. For $D>D_{0}$ we have $\tilde{E}>E$, while for $D<D_{0}$ we have $\tilde{E}<E$. In Figure 9 we plot the substrate concentration $S$, the suspended biomass $u$ and the wall attached biomass $\lambda$ for four different choices of $D_{0}$, along with the data for the corresponding model with $E=$ const as previously. The surface area was kept at $A=A_{\text {reactor }} \approx 0.055 \mathrm{~m}^{2}$ in all simulations. We note that the choice of the detachment rate did not affect the substrate concentration, i.e. it did not affect the prediction of reactor performance. It did, however, affect the biofilm thickness. This is in agreement with [2], a study of biofilm on a porous medium where it was concluded that changes in the mesoscopic detachment rate do not affect the macroscopic reactor performance. On the other hand, larger $D_{0}$, i.e. smaller detachment rates led to thicker biofilms and lower suspended biomass. For larger dilution rates, the detachment rates increased. In contrast to the constant detachment rate, we observed that the biofilm thickness eventually decreased as the dilution rate increased. This did not imply an increase of the suspended biomass for increasing flow rates, which indicates that most of the detached biomass was washed out of the reactor. The observation that a thinner biofilm did not affect the reactor's substrate removal performance indicates that much of the biofilm did not contribute to substrate removal, i.e. that substrate was limited in the inner layers. This is also supported by Figure 10, where we plot the substrate 

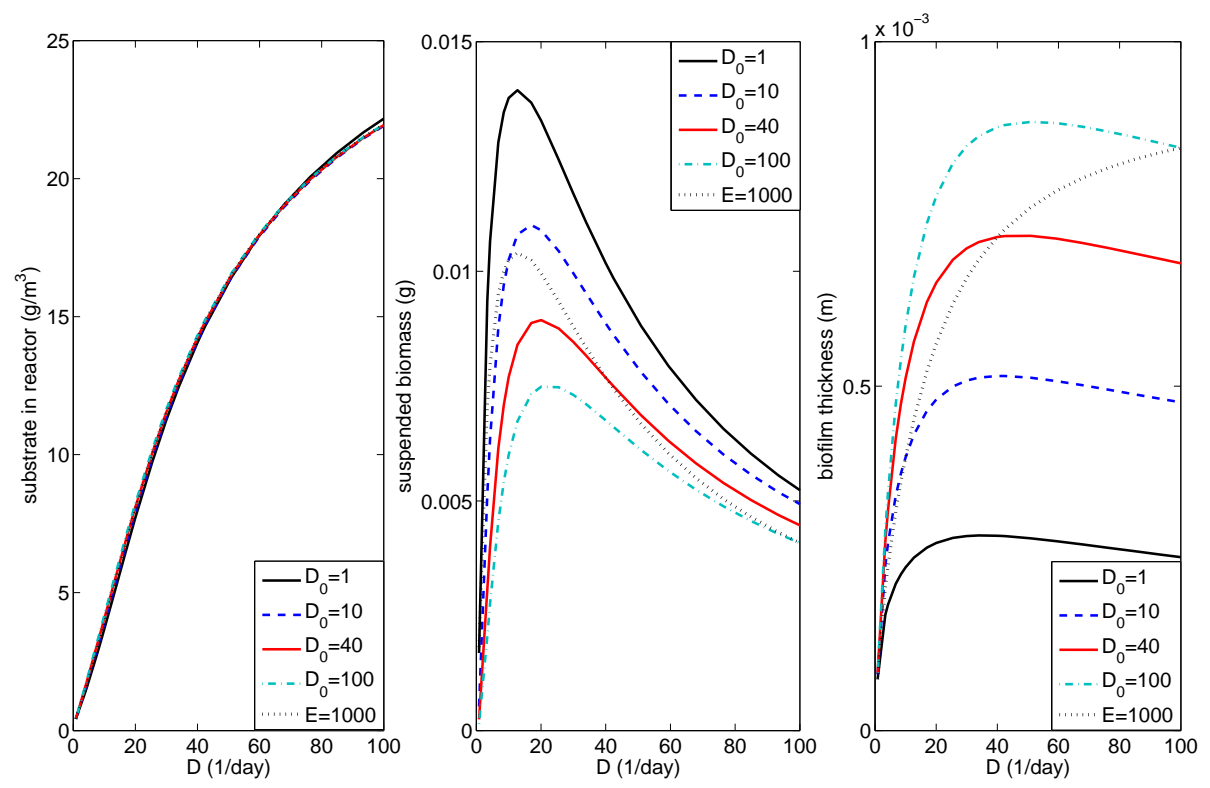

Figure 9: Substrate concentration $S$ (left), suspended biomass $u$ (center) and biofilm thickness $\lambda$ (right) at steady state for dilution rate dependent detachment coefficients as in (37) at $S^{\text {in }}=30 \mathrm{~g} / \mathrm{m}^{3}$.

concentration in the biofilms at steady state attained for the various values of $D_{0}$ at dilution rate $D=20 /$ day. In all four cases the bulk substrate concentration is approximately the same and the substrate concentrations are approximately translations by the difference in biofilm thickness. Thicker biofilms have a thicker inactive inner layer with low substrate concentration.

\subsection{Dependence on reactor parameters}

A possible application of our model is wastewater treatment, where high amounts of biomass are desired. Existing treatment methods use biofilms and suspended biomass (activated sludge) to treat the wastewater. In methods where biofilm is allowed to grow on suspended carriers it is often not known how much the suspended biomass contributes to the removal of unwanted compounds. Often, reactor parameters are designed in such a way that one can assume that most of the suspended biomass is washed out. To investigate this further, we performed numerical simulations of our model varying the reactor parameters $D$ and $A$. The total available colonization 


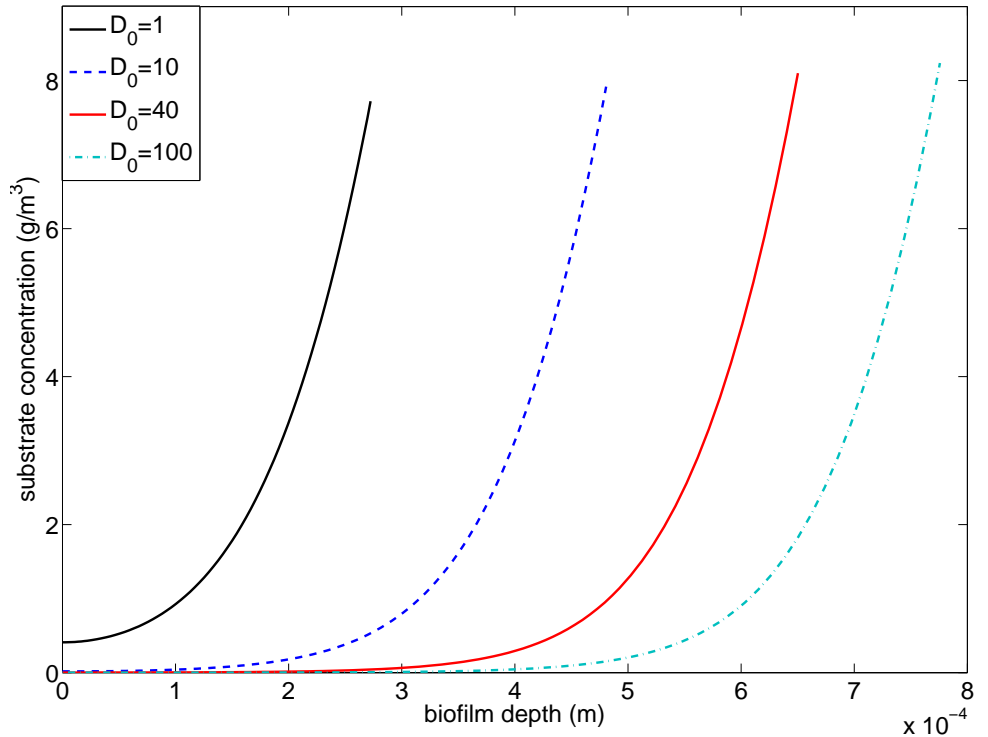

Figure 10: Substrate concentration $C(z)$ within the biofilm at steady state for dilution rate dependent detachment coefficients as in (37) at $S^{\text {in }}=30$ $\mathrm{g} / \mathrm{m}^{3}$ and $D=20 /$ day. The substratum is located to the left and the biofilm-bulk liquid interface to the right in the plot. 

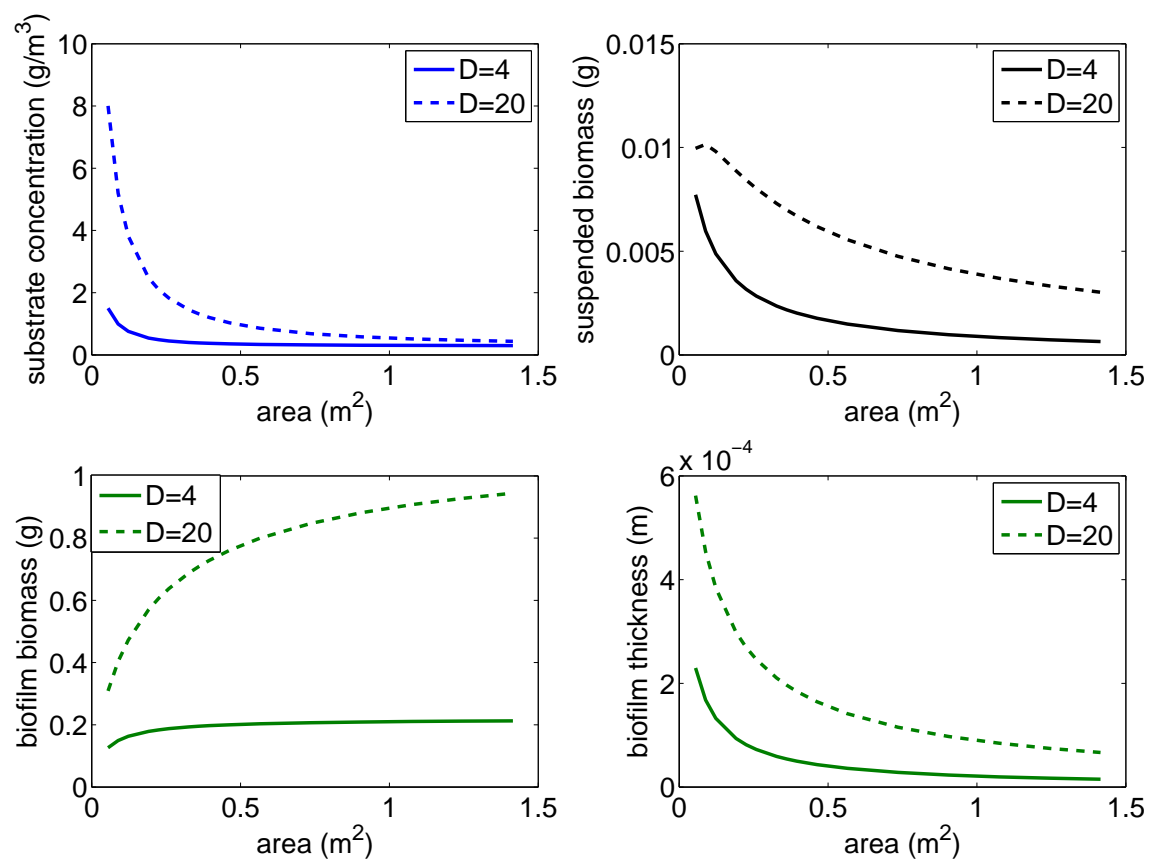

Figure 11: Substrate concentration $S$ (top left), suspended biomass $u$ (top right), biofilm biomass $A \rho \lambda$ (bottom left) and biofilm thickness $\lambda$ (bottom right) at steady state as functions of colonizable area $A$ for dilution rates $D=4$ and $D=20 /$ day at $S^{\text {in }}=30 \mathrm{~g} / \mathrm{m}^{3}$.

area $A$ consists of the reactor's inner walls and bottom and the area provided by a certain amount of suspended carriers. In our simulations we considered an empty reactor with $A \approx 0.055 \mathrm{~m}^{2}$ up to 200 extra carriers with $A \approx 1.4150 \mathrm{~m}^{2}$. Each suspended carrier was assumed to have an area of $0.0068 \mathrm{~m}^{2}$ [17]. The dilution rate $D$ was varied between 1 and 93/day, while $S^{\text {in }}$ was kept at $30 \mathrm{~g} / \mathrm{m}^{3}$.

Higher $D$ resulted in a larger concentration of $S$ in the reactor, due to a higher inflow that transports more substrate into the reactor (see Figure 11). A larger colonization area $A$ contributed to a decrease in substrate concentration due to higher consumption by the increasing biofilm biomass. In fact, even though the biofilm thickness decreased as $A$ increased, the total amount of biofilm biomass increased with $A$. The graph for biofilm biomass in Figure 11 basically reflects the graph for substrate concentration in the reactor.

The behavior of suspended biomass was governed by several forces. For 


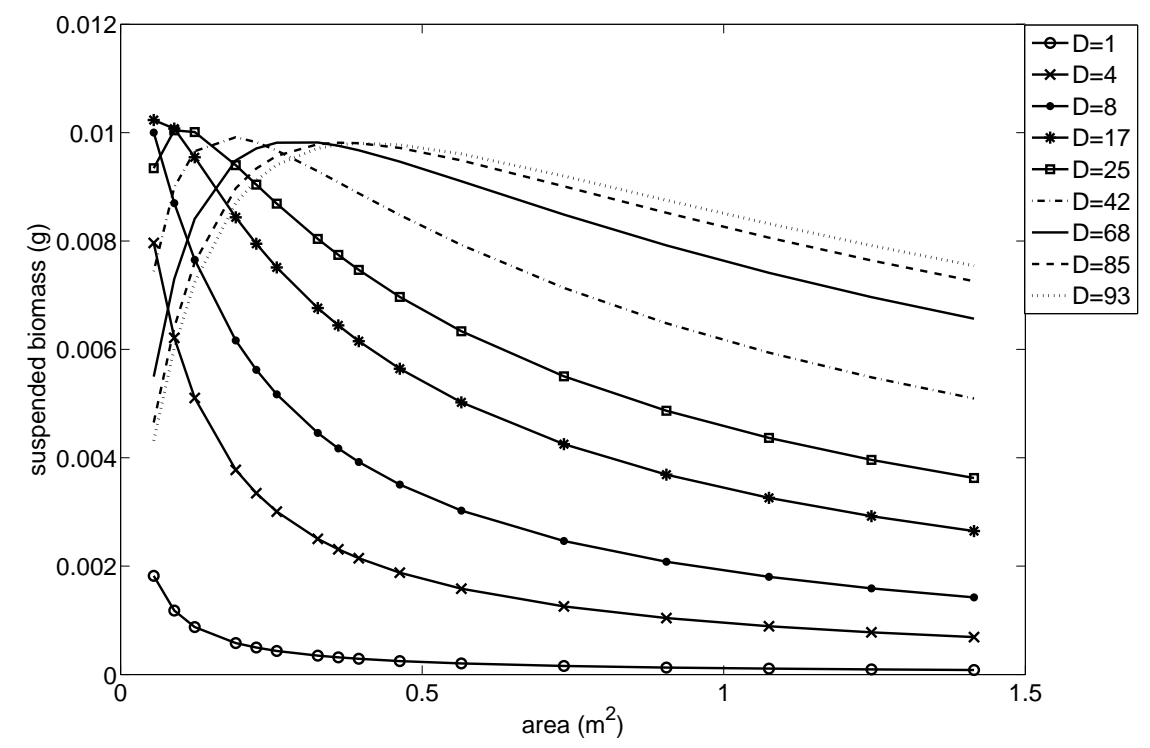

Figure 12: Suspended biomass $u$ at steady state as a function of colonizable area $A$ for different dilution rates $D$ at $S^{i n}=30 \mathrm{~g} / \mathrm{m}^{3}$.

small dilution rates the amount of suspended biomass decreased as the surface area for colonization increased. The increase of $A$ resulted in a larger biofilm biomass (see Figure 11), which in turn limited the substrate, thereby causing a decrease in suspended biomass due to substrate limitation. Initially this decrease was rapid but leveled off as $A$ increased. For larger dilution rates, the suspended biomass increased for small surface areas but then declined and plateaued (see Figure 12). The increasing dilution rate $D$ brought more substrate to the reactor, but it also increased washout of biomass. The peak value for suspended biomass decreased and was attained at larger values of $A$ as $D$ increased. In all cases, the plateaued value for suspended biomass increased with the dilution rate. Since the biofilm biomass increased with $A$ and $D$, there was a larger contribution of biomass to the bulk liquid through erosion as both $A$ and $D$ increased. The washout effect was smaller for larger $A$ due to the larger contribution of biomass from the biofilm, balancing out the washout. In summary, suspended biomass increased with $D$ and decreased with $A$, except for low ranges of $A$ where the washout effect of higher $D$ was noticeable.

Generally we observe that increasing dilution rate increased the substrate removal efficiency which is easily calculated through $Q\left(S^{i n}-S^{*}\right)$ [g/day], 


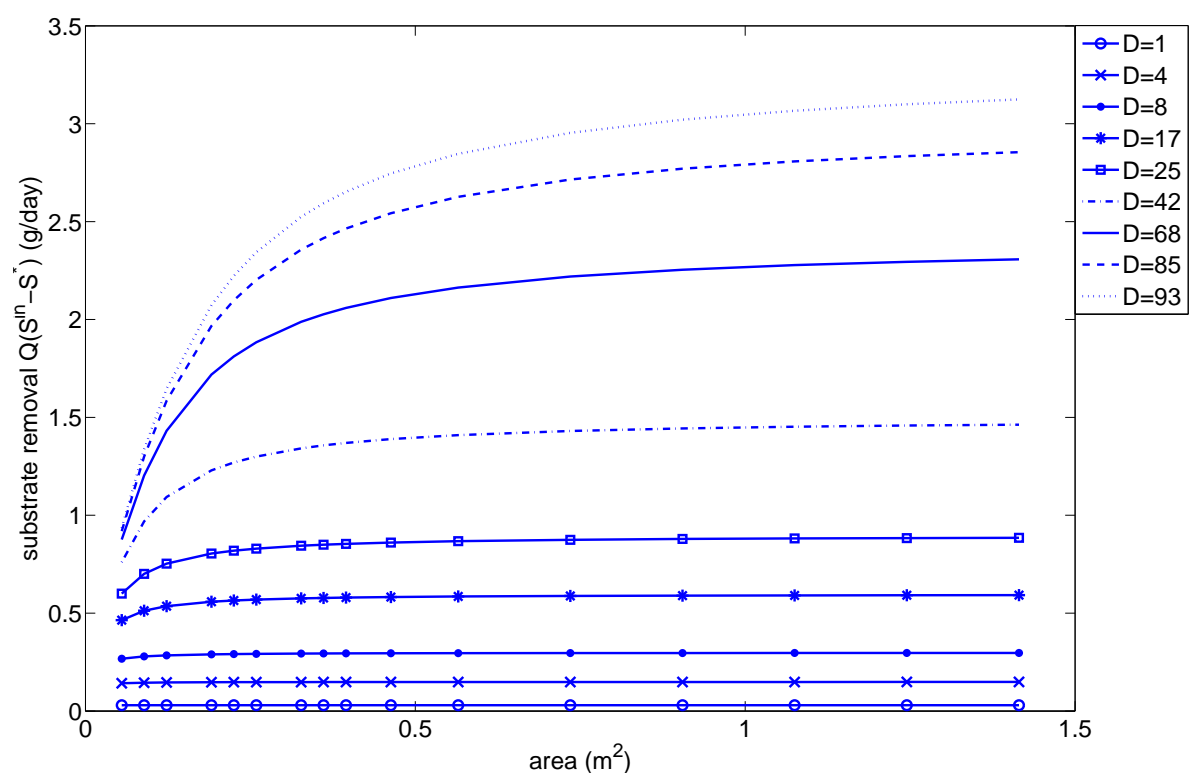

Figure 13: Substrate removal at steady state as a function of colonizable area $A$ for different dilution rates $D$ at $S^{i n}=30 \mathrm{~g} / \mathrm{m}^{3}$.

where $S^{*}$ is the steady state substrate concentration. For small dilution rates, $D<\mu_{u}^{\max }$, the surface colonization rate had no effect on the substrate removal rate. However, as $D$ became larger than the growth rate, i.e. as the washout of suspended bacteria began to dominate growth, the substrate removal rate increased initially as $A$ increased and then leveled off for larger $A$. The bigger the dilution rate the more pronounced was this effect and the later the leveling off occurred (see Figure 13). Interestingly, by increasing $A$ we could increase the overall removal but were not able to decrease the final concentration of $S$ in the effluent beyond a lower limit, regardless of the dilution rate. Hence, addition of suspended carriers would improve removal (significantly for higher $D$ ) to a certain extent.

We denote the contribution of suspended biomass to the overall substrate removal by $\phi$ and calculate it through

$$
\phi=\frac{\frac{u^{*} \mu_{u}\left(S^{*}\right)}{\gamma}}{Q\left(S^{i n}-S^{*}\right)}=\frac{u^{*} \mu_{u}^{\max } S^{*}}{\gamma Q\left(S^{i n}-S^{*}\right)\left(K_{u}+S^{*}\right)}
$$

where $u^{*}$ denotes the steady state suspended biomass. $\phi$ decreased at all dilution rates as the colonization area increased, indicating that the biofilm dominated as the main actor in substrate removal (see Figure 14). However, 

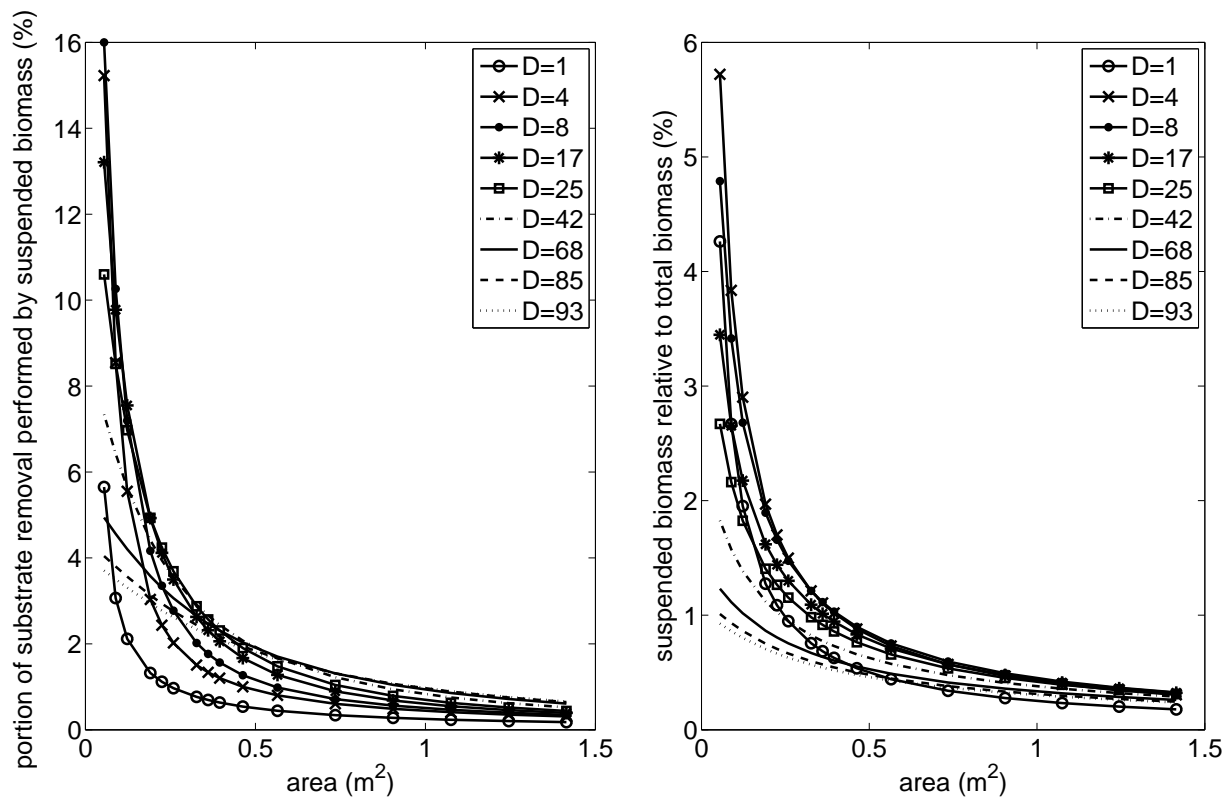

Figure 14: Percentage of substrate removal performed by suspended biomass (left) and amount of suspended biomass relative to total biomass (right), at steady state as a function of colonizable area $A$ for different dilution rates $D$ at $S^{i n}=30 \mathrm{~g} / \mathrm{m}^{3}$.

the suspendeds contributed to the removal significantly in medium ranges of $D$ and low colonization areas, although never reaching beyond $16 \%$. This indicates that the suspended biomass can make a considerable contribution to reactor performance, even if the amount of suspendeds is small compared to the amount of biofilm. In fact, suspended biomass was relatively more efficient in substrate removal than the biofilm. This is due to the fact that in biofilms the bacteria in the inner layers often, particularly in thick biofilms, live under nutrient limitations and do not contribute greatly to substrate degradation. $\phi$ corresponded to the behavior of the suspended biomass for the different dilution rates, i.e. it increased initially in the lower ranges of $D$ until it reached a peak and subsequently decreased due to higher washout.

\section{Conclusion}

In this paper we studied a mathematical model of bacterial population and resource dynamics in a CSTR with wall attachment. This is a modification 
of Freter's model, in which we treat the wall attached bacteria as biofilms. The resulting mathematical model is more complex than the original Freter model, because the reactor mass balance is coupled with a diffusion-reaction equation for the substrate in the biofilm. The added algebraic and physical complexity did not add increased dynamic complexity. The stability conditions for the trivial equilibrium of the modified model are essentially the same as for the original Freter model. If the trivial equilibrium is unstable, the system attains a non-trivial equilibrium, in which both suspended and biofilm biomass co-exist. Overall, the model shows a preference of the biomass for the biofilm mode of growth, as also observed in natural systems. In particular for large reactor flow rates suspended biomass is washed out faster than it can reproduce.

Numerical simulations of the model highlight that changes in the detachment rate affect the biofilm thickness, but do not affect the overall reactor performance.

Although the amount of suspended biomass is small compared to the biomass accumulated in the biofilm, its contribution to the reactor's substrate removal performance is significant in medium ranges of the dilution rate and small colonization areas. In fact, it appears that suspended biomass is relatively more efficient than the biofilm. This can be explained by the fact that in the deeper layers of the biofilm substrate can become limited, wherefore the bacteria there do not contribute to the removal process greatly.

In engineering applications, the performance of a reactor is often increased by increasing the colonizable surface area on which biofilms can grow. Our model reflects that this indeed increases reactor performance, but only to a certain extent, after which the performance levels off, as we observed a lower threshold for the steady-state bulk substrate concentration. In general, the higher the reactor flow rate and thus the substrate supply, the more is gained by providing additional surface area for biofilm formation.

While our study was motivated by wastewater engineering applications, we should point out that the mathematical model used here is too simplified to be quantitative. On the other hand, complicated engineering models, such as the International Water Association's Activated Sludge Models [13] or the Anaerobic Digestion Model [14] are much too involved and depend on too many parameters to be accessible for qualitative studies. Under this light, focusing on simplified and idealized scenarios can be a useful first step toward a mathematical and qualitative understanding of more involved systems $[11,12,18,20]$. Moreover, they can be the starting point for targeted computational studies of more involved models. This is the next step in our 
research program.

\section{Nomenclature}

A

$a_{u}$

$a_{w}$

$D$

$D_{c}$

E

F

$K_{\lambda}$

$K_{u}$

$k_{\lambda}$

$k_{u}$

$k_{w}$

$m_{u}$

$m_{w}$

$Q$

$S$

$S^{\text {in }}$

$u$

$u$

V

$w$

$w_{\max }$

$W=w / w_{\max }$

Greeks

$\alpha \quad$ attachment rate

$\beta$

$\lambda$

$\mu_{\lambda}^{\max }$

$\mu_{u}^{\max }$

$\gamma$

$\rho$

area

dilution rate

biofilm thickness

yield half-saturation Monod const. of suspended bacteria (Freter)

$\left(\mathrm{m}^{2}\right)$

half-saturation Monod const. of wall-attached bacteria (Freter) $\left(\mathrm{g} / \mathrm{m}^{3}\right)$

diffusion coefficient

erosion parameter

flow (velocity) through the reactor (Freter)

half-saturation Monod constant of biofilm

half-saturation Monod constant of suspended bacteria

death rate of biofilm

death rate of suspended bacteria

death rate of wall-attached bacteria in Freter model

maximum growth rate of suspended bacteria (Freter)

maximum growth rate of wall-attached bacteria (Freter)

flow (velocity) through the reactor

substrate concentration

substrate concentration at inlet

suspended bacteria

concentration of suspended bacteria (Freter)

volume of the reactor

areal biomass density of wall-attached bacteria

maximum areal biomass density of wall-attached bacteria

wall occupancy fraction

$(1 /$ day $)$

$\left(\mathrm{m}^{2} /\right.$ day $)$

$(1 / \mathrm{m} \cdot$ day $)$

$\left(\mathrm{m}^{3} /\right.$ day $)$

$\left(\mathrm{g} / \mathrm{m}^{3}\right)$

$\left(\mathrm{g} / \mathrm{m}^{3}\right)$

$(1 /$ day $)$

$(1 /$ day $)$

$(1 /$ day $)$

$(1 /$ day $)$

$(1 /$ day $)$

$\left(\mathrm{m}^{3} /\right.$ day $)$

$\left(\mathrm{g} / \mathrm{m}^{3}\right)$

$\left(\mathrm{g} / \mathrm{m}^{3}\right)$

(g)

$\left(\mathrm{g} / \mathrm{m}^{3}\right)$

$\left(\mathrm{m}^{3}\right)$

$\left(\mathrm{g} / \mathrm{m}^{2}\right)$

$\left(\mathrm{g} / \mathrm{m}^{2}\right)$

$(-)$

$(1 /$ day $)$

$(1 /$ day $)$

(m)

$(1 /$ day $)$

$(1 /$ day $)$

maximum growth rate of suspended bacteria

$(-)$

biofilm biomass density

\section{References}

[1] F. Abbas and H.J. Eberl, (2011). Analytical flux approximation for the Monod boundary value problem. Appl. Math. Comp. 218(4):1484-1494. 
[2] F. Abbas and H.J. Eberl, (accepted). Investigation of the role of mesoscale detachment rate expressions in a macroscale model of a porous medium biofilm reactor. Int. J. Biomath. Biostats. [scheduled for 2(1), 2011]

[3] F. Abbas, R. Sudarsan and H.J. Eberl, (accepted). Longtime behavior of onedimensional biofilm models with shear dependent detachment rates. Math. Biosc. Eng.

[4] M.M. Ballyk, D.A. Jones, and H.L. Smith, (2001). Microbial competition in reactors with wall attachment. Microbial Ecology 41(3):210-221.

[5] M.M. Ballyk, D.A. Jones, and H.L. Smith, (2008). The biofilm model of Freter: a review. In P. Magal, S. Ruan (eds), Structured population models in biology and epidemiology, Springer Lecture Notes in Mathematics Vol.1936.

[6] E. Bester, E.A. Edwards and G.M. Wolfaardt, (2009). Planktonic cell yield is linked to biofilm development. Can. J. Microbiology, 55(10):1195-1206.

[7] B. Boldin, (2008). Persistence and spread of gastro-intestinal infections: the case of enterotoxigenic Escherichia coli in piglets. B. Math. Biol., 70(7):2077-2101.

[8] J.W. Costerton, Z. Lewandowski, D.E. Caldwell, D.R. Korber and H.M. LappinScott, (1995). Microbial Biofilms. Ann. Rev. Microbiology 49:711-745.

[9] H.C. Flemming, (2000). Biofilme - das Leben am Rande der Wasserphase Nachr. Chemie 48:442-447.

[10] R. Freter, H. Brickner, J. Fekete, M. Vickerman and K. Carey, (1983). Survival and implantation of Escherichia coli in the intestinal tract. Infect. Immun. 39:686-703.

[11] E.V. Grigorieva and E.N. Khailov, (2010). Minimization of pollution concentration on a given time interval for the waste water cleaning plant. J. Control Sci. Eng. vol. 2010, Article ID 712794, 10 pages.

[12] M. El Hajji, F. Mazenc, and J. Harmand, (2010). A mathematical study of a syntrophic relationship of a model of anaerobic digestion process. Math. Biosci. Eng. 7(3):641-656.

[13] M. Henze, W. Gujer, M. Takashi, and M. van Loosdrecht, (2002). Activated Sludge Models ASM1, ASM2, ASM2d and ASM3. IWA Publishing.

[14] IWA Task Group, (2002). Anaerobic Digestion Model No. 1 (ADM1). IWA Publishing.

[15] D. Jones, H. V. Kojouharov, and D. Le, H. Smith, (2003). The Freter model: A simple model of biofilm formation. Math. Bio. 4\%:137-152.

[16] Z. Lewandowski and H. Beyenal, (2007). Fundamentals of biofilm research. CRC Press, Boca Raton. 
[17] A. Masic, J. Bengtsson, and M. Christensson, (2010). Measuring and modeling the oxygen profile in a nitrifying Moving Bed Biofilm Reactor. Math. Biosci. 22\%:1-11.

[18] J. Moreno, (1999). Optimal time control of bioreactors for the wastewater treatment. Optim. Control Appl. Meth. 20:145-164.

[19] E. Morgenroth, (2003). Detachment: an often-overlooked phenomenon in biofilm research and modeling. In: S. Wuertz et al (eds), Biofilms in Wastewater Treatment, pp 246-290, IWA Publishing, London.

[20] T.G. Müller, N. Noykova, M. Gyllenberg, and J. Timmer, (2002). Parameter identification in dynamical models of anaerobic waste water treatment. Math. Biosci. 17r7-178:147-160.

[21] L.A. Pritchett and J.D. Dockery, (2001). Steady state solutions of a onedimensional biofilm model. Math. Comput. Model. 33:255-263.

[22] B.E. Rittmann, (1982). The effect of shear stress on biofilm loss rate. Biotech. Bioeng. 24:501-506.

[23] B.E. Rittmann and P.L. McCarty, (2001). Environmental Biotechnology. McGraw-Hill.

[24] H.L. Smith and P. Waltman, (1995). The theory of the chemostat. Cambridge University Press, Cambridge, UK.

[25] E.D. Stemmons and H.L. Smith, (2000). Competition in a chemostat with wall attachment. SIAM J. Appl. Math. 61(2):567-595.

[26] P.S. Stewart, (2003). Diffusion in biofilms. J. Bacteriol. 185(5):1485-1491.

[27] B. Szomolay, (2008). Analysis of a moving boundary value problem arising in biofilm modeling. Math. Meth. Appl. Sci. 31:1835-1859.

[28] W. Walter, (2000). Gewöhnliche Differentialgleichungen. 7th ed, SpringerVerlag, Berlin.

[29] O. Wanner and W. Gujer, (1986). A multispecies biofilm model. Biotech. Bioeng. 28:314-328.

[30] O. Wanner, H. Eberl, E. Morgenroth, D.R. Noguera, C. Picioreanu, B. Rittmann, and M. van Loosdrecht, (2006). Mathematical modeling of biofilms, Scientific and Technical Report No.18. IWA Publishing.

[31] J.B. Xavier, C. Picioreanu, and M.C.M. van Loosdrecht, (2004). A modeling study of the activity and structure of biofilms in biological reactors. Biofilms 1(4):377-391. 